\title{
Hydrothermal Synthesis of Calcium Sulfate Whisker from Flue Gas Desulfurization Gypsum
}

\author{
LIU Chengjun (刘承军), ZHAO Qing (赵青)**, WANG Yeguang (王野光), SHI Peiyang (史培阳), JIANG \\ Maofa (姜茂发)
}

Key Laboratory for Ecological Metallurgy of Multimetallic Ores (Ministry of Education), Northeastern University, Shenyang 110819, China

Abstract Plenty of flue gas desulfurization (FGD) gypsum generated from coal-fired power plants for sulfur

dioxide sequestration caused many environmental issues. Preparing calcium sulfate whisker (CSW) from FGD

gypsum by hydrothermal synthesis is considered to be a promising approach to solve this troublesome problem and

utilize calcium sulfate in a high-value-added way. The effects of particle size of FGD gypsum, slurry concentration,

and additives on CSW were investigated in this work. The results indicated that fine particle size of FGD gypsum

and moderately high slurry concentration were beneficial for crystal nucleation and growth. Three additives of

magnesium chloride, citric acid, and sodium dodecyl benzene sulfonate (SDBS) were employed in this study. It was

found that mean length and aspect ratio of CSW were both decreased by the usage of magnesium chloride, while a

small quantity of citric acid or SDBS could improve the CSW morphology. When multi-additives of citric

acid-SDBS were employed, the mean length and aspect ratio increased more than $20 \%$. Moreover, surface

morphology of CSW went better, and the particle size and crystal shape became more uniform.

*Supports by the Natural Science Foundation of China (No. 51374059, No. 51304042), and the Fundamental Research Funds for The Central Universities of China (No. N130402020).

**To whom correspondence should be addressed. E-mail: qingzhao0121@ gmail.com. 
Keywords calcium sulfate whisker, hydrothermal, synthesis, environment

\section{INTRODUCTION}

Flue gas desulfurization (FGD) processes using calcium-bearing absorbents have been adopted by about $90 \%$

of coal-fired power plants in China for sulfur dioxide sequestration because of its high desulfurisation performance and low operating cost. Million tons of calcium sulfate-rich FGD gypsum, the principle solid by-product, was generated and sent to landfills for its disposal per year since 2009, which caused many environmental concerns [1,

2]. The properties of FGD gypsum were proved to be comparable to that of natural gypsum [3], so the utilization of FGD gypsum in a high-value-added way has attracted much interest recent decades [4-6]. Preparing calcium sulfate whisker (CSW) from FGD gypsum by hydrothermal synthesis is a promising approach to treat FGD gypsum and recover the valuable calcium sulfate, while little attention has far been paid on it [7]. CSW, an excellent inorganic material, is widely used in rubber and bituminous industry, friction material manufacture, and environmental engineering to improve products mechanical properties $[8,9]$. In contact with crystallization water, three phases of CSW can crystallize: calcium sulfate dihydrate $(\mathrm{DH})$ whisker, calcium sulfate hemihydrate $(\mathrm{HH})$ whisker, and calcium sulfate anhydrous (AH) whisker. Common structures in all CSW phases are chains in the form of 
[-Ca-SO $\left.4_{4}-\mathrm{Ca}-\mathrm{SO}_{4}-\right]$, where sulfate tetrahedra are coordinated through oxygen atoms with two neighboring calcium ions in chain direction, and this crystal structure contains "channels" that can accommodate variable amounts of water [10]. During the hydrothermal synthesis process, some DH, the only phase of calcium sulfate in FGD gypsum, firstly dissolves in aqueous releasing $\mathrm{Ca}^{2+}$ and $\mathrm{SO}_{4}^{2-}$ ions, and then when concentrations of $\mathrm{Ca}^{2+}$ and $\mathrm{SO}_{4}^{2-}$ reach supersaturation HH nucleuses could be generated and gradually develop into needle-like whisker because of the different growth rates in various directions. A transformation from $\mathrm{HH}$ whisker into AH whisker could occur if process temperature is enough high (normally higher than $170^{\circ} \mathrm{C}$ ).

The physical properties of CSW, such as packing density, filtration rate, and mechanical strength, are highly depend on its morphology, and the morphology has been reported to be sensitive to some processing conditions such as temperature, duration, $\mathrm{pH}$ value of solution, and solution composition [11-13]. However, few articles focused on the influences of other experimental factors like particle size of raw materials, slurry concentration (mass ratio between FGD gypsum and deionized water), and chemical additives on CSW in hydrothermal synthesis process have been published. With respect to studies addressing calcium sulfate crystal preparation, it was known that inorganic or organic additives, act as retarders, accelerators, thickeners, plasticizers, or for air entrainment or as deflocculating agents, could affect the morphology of crystals [14-16]. Some ions incorporate into crystals or absorb on certain crystal faces during the calcium sulfate crystal growth process, so the surface energy and growth rates in different directions could be changed [17-19]. Wang et al. [20] prepared CSW from natural gypsum by 
hydrothermal synthesis method, and reported that the aspect ratio, the ratio between length and diameter, increased from 30 to higher than 50 by adding magnesium chloride additive. However, Yang et al. [21] did not find notable improvement in CSW morphology when tests conducted with higher slurry concentration. Moreover, some organic reagents like citric acid [22, 23] and sodium dodecyl benzene sulfonate (SDBS) [24] were usually used in calcium sulfate preparation under atmospheric condition to control calcium sulfate crystal nucleation and growth processes, while the investigation concerning the influence of these additives on morphology of CSW in hydrothermal synthesis process has yet to be revealed.

Aiming at the manufacture of excellent performance CSW from FGD gypsum, the efforts of particle size of FGD gypsum, slurry concentration, and additives, on CSW morphology were investigated in the current work. In the other part of this study [25], the synthesized CSW was surface modified to improve its hydrophobic property for the application in polymeric composites preparation.

\section{EXPERIMENTAL}

\subsection{Materials}

FGD gypsum used in this experimental investigation was taken from a Chinese steel company. Inductively

coupled plasma-optical emission spectrometry (ICP-OES) analysis was carried out by Varian Vista-MPX against suitably calibrated standards ( $5 \sim 20 \mathrm{ppm}$ ) on aqueous extracts from $100 \mathrm{mg}$ samples diluted to $500 \mathrm{ml}$ to detect the 
chemical composition of the FGD, and the results are given in Table 1. Additives of magnesium chloride ( > 99.7wt.\%), citric acid ( > 99.5wt.\%), and SDBS ( > 99.0wt.\%) were employed for CSW morphology control. Some chemical reagents of magnesium sulfate and calcium chloride anhydrous were also used as raw materials to prepare

CSW for a comparison.

Table 1 Chemical composition of raw FGD gypsum (wt.\%)

\begin{tabular}{cccccccc}
\hline $\mathrm{CaO}$ & $\mathrm{SiO}_{2}$ & $\mathrm{Al}_{2} \mathrm{O}_{3}$ & $\mathrm{SO}_{3}$ & $\mathrm{MgO}$ & $\mathrm{Fe}_{2} \mathrm{O}_{3}$ & $\mathrm{H}_{2} \mathrm{O}$ & Others \\
\hline 31.9 & 2.6 & 0.7 & 42.5 & 0.9 & 0.8 & 11.0 & 9.6 \\
\hline
\end{tabular}

\subsection{Methods}

FGD gypsum was poured into a jar placed in a planetary mill, and then started the mill with a revolving speed of

$150 \mathrm{r} \cdot \mathrm{min}^{-1}$ and maintained for various durations to obtain raw materials with different particle sizes.

Brunauer-Emmett-Teller adsorption analysis was conducted to detect specific surface area of the milled samples,

and BT-9300S laser particle size analyzer was employed to determine their particle size. $10 \mathrm{~g}$ FGD gypsum powder

and some deionized water was mixed in a glass beaker by a magnetic stirrer at room temperature, after which the $\mathrm{pH}$

value of this slurry was adjusted to 3 by adding dilute sulfate acid. Some magnetic impurities could be absorbed and

removed from the FGD gypsum in this process. High-pressure reactor illustrated by Fig. 1 was used in this study for 
hydrothermal synthesis of CSW. FGD gypsum slurry and additive were both poured into the reactor body, and then started heating system and agitation. After $2 \mathrm{~h}$ thermal insulation at $140{ }^{\circ} \mathrm{C}$ (the optimum conditions obtained from previous study), filtration and washing were carried out to separate hydrothermal synthesis products from filtrate, and CSW were obtained after a drying treatment at $100^{\circ} \mathrm{C}$ for $3 \mathrm{~h}$. CSW morphology was studied by OLYCIA P3 high-resolution optical microscope and scanning electron microscopy (SEM), and the length and diameter of 200 CSW in every sample was measured to calculate the mean length and aspect ratio. X-ray diffraction (XRD) analysis was also employed for determining phase composition of products. A flow sheet of the process route is given in Fig.

2.

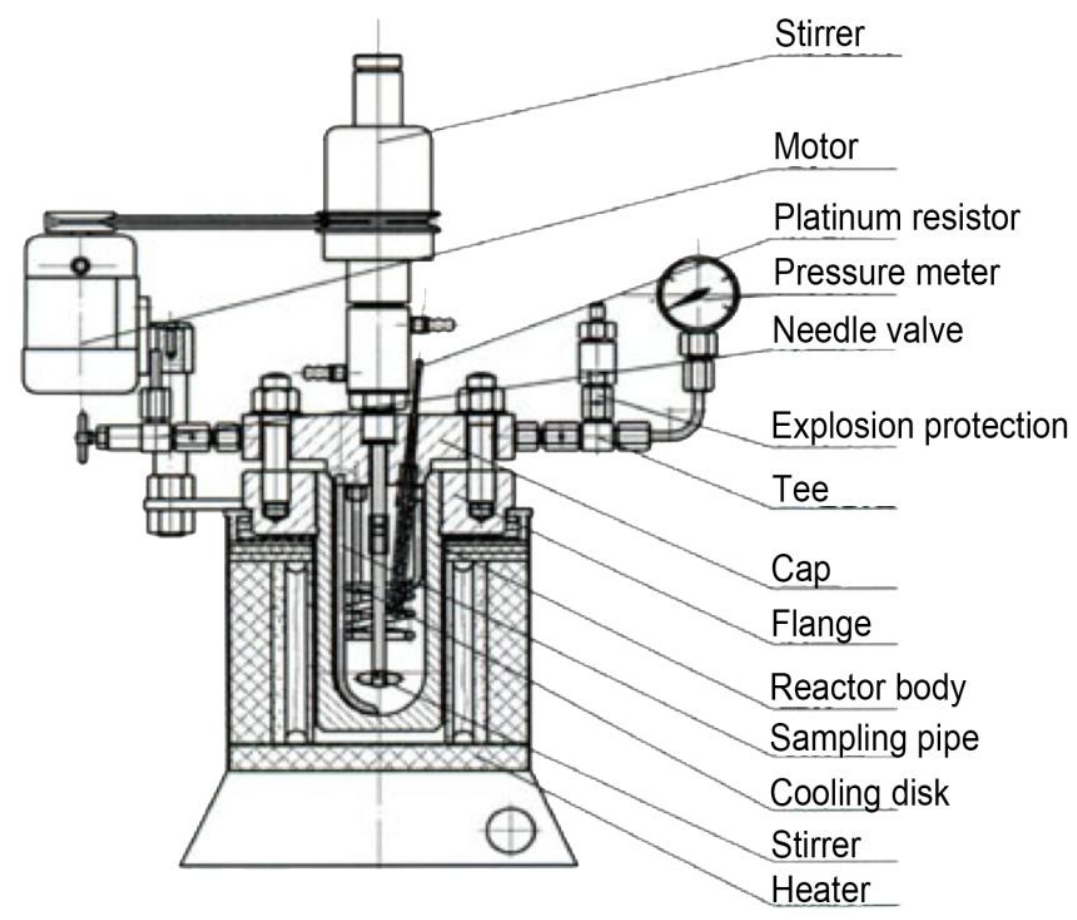

Figure 1 Illustration of the high-pressure reactor used in this study. 


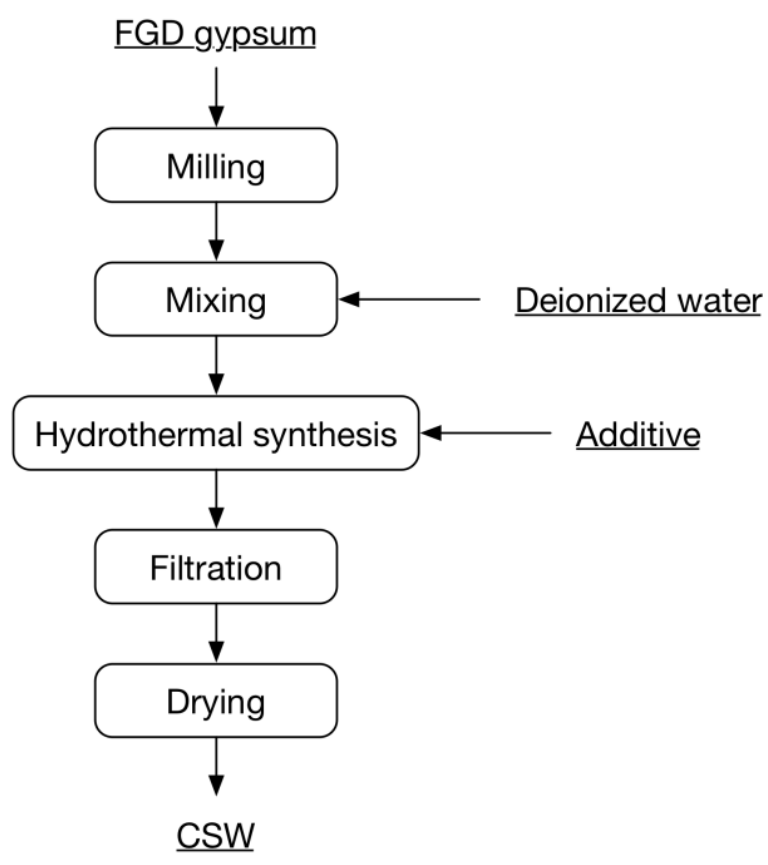

Figure 2 Flow sheet of the process route.

\section{RESULTS AND DISCUSSION}

Effects of particle size of FGD gypsum and slurry concentration on CSW morphology were investigated without using additive, and the various additives were then studies under the reasonable conditions obtained from following research.

\subsection{Effect of particle size of FGD gypsum}

As it has been mentioned above, in the initial phase of hydrothermal synthesis process of CSW, some DH in FGD gypsum dissolves in aqueous followed by the CSW precipitation when the concentrations of $\mathrm{Ca}^{2+}$ and $\mathrm{SO}_{4}^{2-}$ ions reach supersaturation. Therefore, it could be reasonably speculated that the crystallization process of CSW can 
be improved by some factors that could promote dissolution rate of $\mathrm{DH}$. According to this view, the effect of particle size of FGD gypsum on CSW morphology was investigated below.

Particle size of FGD gypsum powders was detected using laser particle size analyzer after mill treatment with different durations ( $0 \mathrm{~min}, 60 \mathrm{~min}, 120 \mathrm{~min}$, and $240 \mathrm{~min}$ ), and the results are given in Fig. 3. It was found that the FGD gypsum powder without milling treatment to be mostly in the range of $40 \sim 80 \mu \mathrm{m}$, and the size of particles gradually decreased with the milling duration. Nearly a half of FGD gypsum was smaller than $30 \mu \mathrm{m}$ after 120 min milling, and about $61 \%$ of powder was smaller than $10 \mu \mathrm{m}$ when milling duration went to $240 \mathrm{~min}$. $D_{50}$ known as the median diameter or the medium value of the particle size distribution was used in this study for characterizing of particle size. They are the values of the particle diameter at 50\% in the cumulative distribution, which were 37.24 $\mu \mathrm{m}, 30.83 \mu \mathrm{m}, 20.94 \mu \mathrm{m}$, and $6.15 \mu \mathrm{m}$ when the milling duration were $0 \mathrm{~min}, 60 \mathrm{~min}, 120 \mathrm{~min}$, and $240 \mathrm{~min}$. 

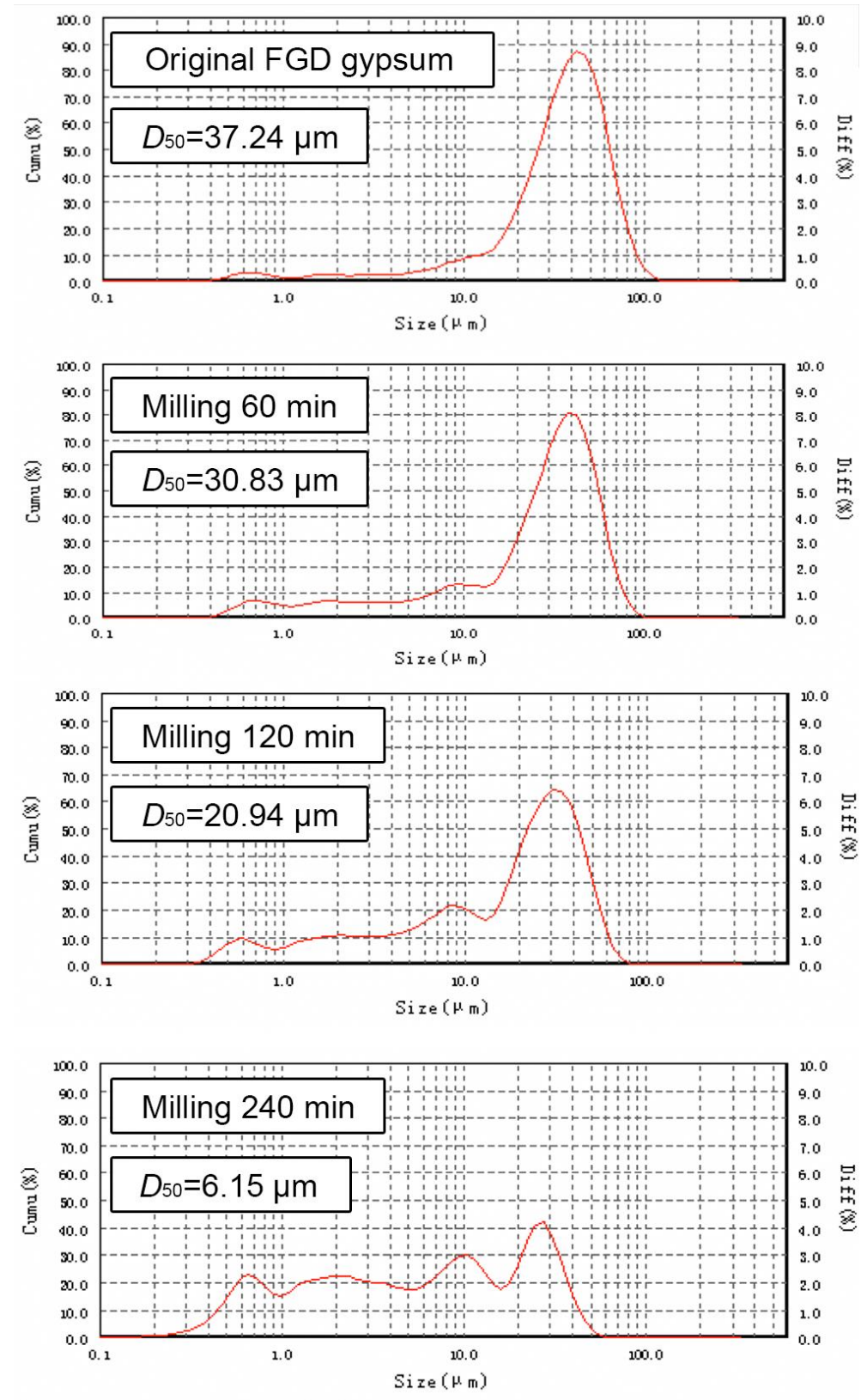

Figure 3 Particle size distribution of the FGD gypsum prepared for different milling durations.

The relationship between $D_{50}$ and specific surface area of FGD gypsum was studied, showing a notable elevation of specific surface area with particle size reduction. The dissolution process of FGD gypsum would be promoted by this change, and the crystal growth rate of CSW may consequently increase. A batch of hydrothermal experiments with various $D_{50}$ s of FGD gypsum was carried out in slurry with a same concentration of $14.28 \%$. The 
morphology of products obtained in this part was investigated using OLYCIAP3 high-resolution optical microscope,

and some microscope images are given in Fig. 4.
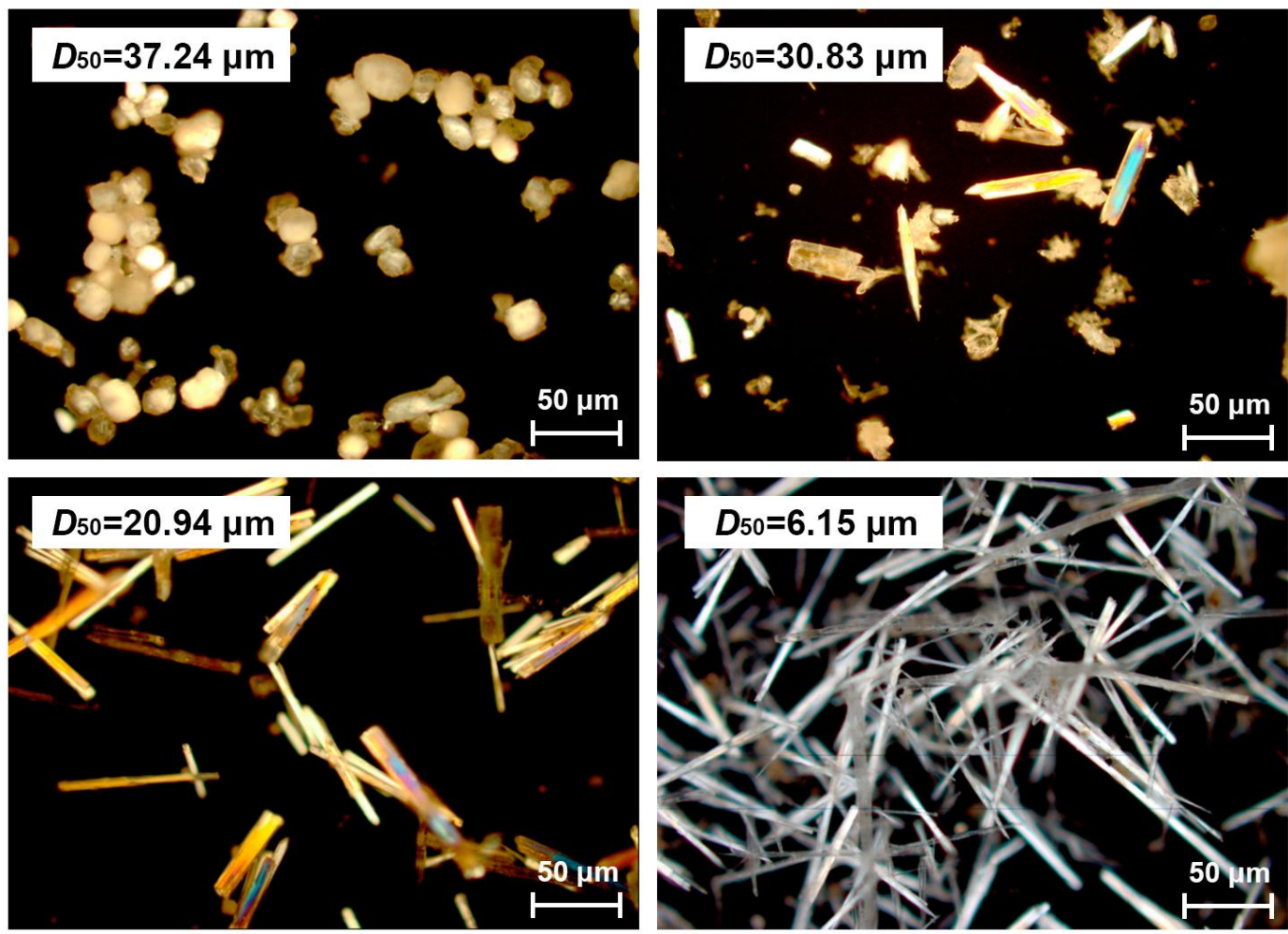

Figure 4 Microscope images of products obtained from different FGD gypsum sizes.

Results demonstrated that the particle size of FGD gypsum played a pivotal role on the morphology of product.

The crystal prepared from $D_{50}=37.24 \mu \mathrm{m}$ test (without mill treatment) was shown to be spherical or spherical-like,

and no needle-like CSW could be found in the microscope image. Moreover, phase composition of this product was

determined by XRD analysis, showing the principal phase to be raw material of DH. With this particle size of FGD

gypsum, dissolution rate of DH was too slow to offer adequate $\mathrm{Ca}^{2+}$ and $\mathrm{SO}_{4}^{2-}$ ions for $\mathrm{CSW}$ crystallization. When 
the particle size went down, needle-like CSW formed and the length significantly increased, which would be clearly

observed in Fig. 4. After statistics and calculation, it was also found that the aspect ratio reached about 53 when particle size decreased to $D_{50}=6.15 \mu \mathrm{m}$. In the tests using fine FGD gypsum, $\mathrm{Ca}^{2+}$ and $\mathrm{SO}_{4}^{2-}$ quickly dissolved in aqueous leading to a supersaturation, so more CSW nucleus produced and grew fast in slurry. Therefore, in the hydrothermal synthesis process, the reduction of particles size of FGD gypsum effectively facilitated CSW crystallization and improved its morphology, but the cost of crushing will naturally simultaneously increase.

\subsection{Effect of slurry concentration}

A series of tests with constant particle size of FGD gypsum $\left(D_{50}=6.15 \mu \mathrm{m}\right)$ and various slurry concentrations $(9.09 \%, 11.11 \%, 14.28 \%$, and $20.00 \%)$ was carried out. The morphology of products was investigated, and some microscope images are shown in Fig. 5. Observation results revealed that CSW formed in all tests, while the CSW prepared from different slurry concentrations exhibited various morphologies. The longest CSW with high uniformity was obtained when the slurry concentration was $14.28 \%$. In order to make more meaningful comparisons between the CSW morphology prepared from different slurry concentrations, the mean length and aspect ratio of the four products were calculated, and the results can be seen in Fig. 6. It was indicated that the two indexes had a similar trend that both increased with slurry concentration below $14.28 \%$ and sharply decreased when the concentration rose higher. With the reasonable slurry concentration (14.28\%) of this study, the mean length of 

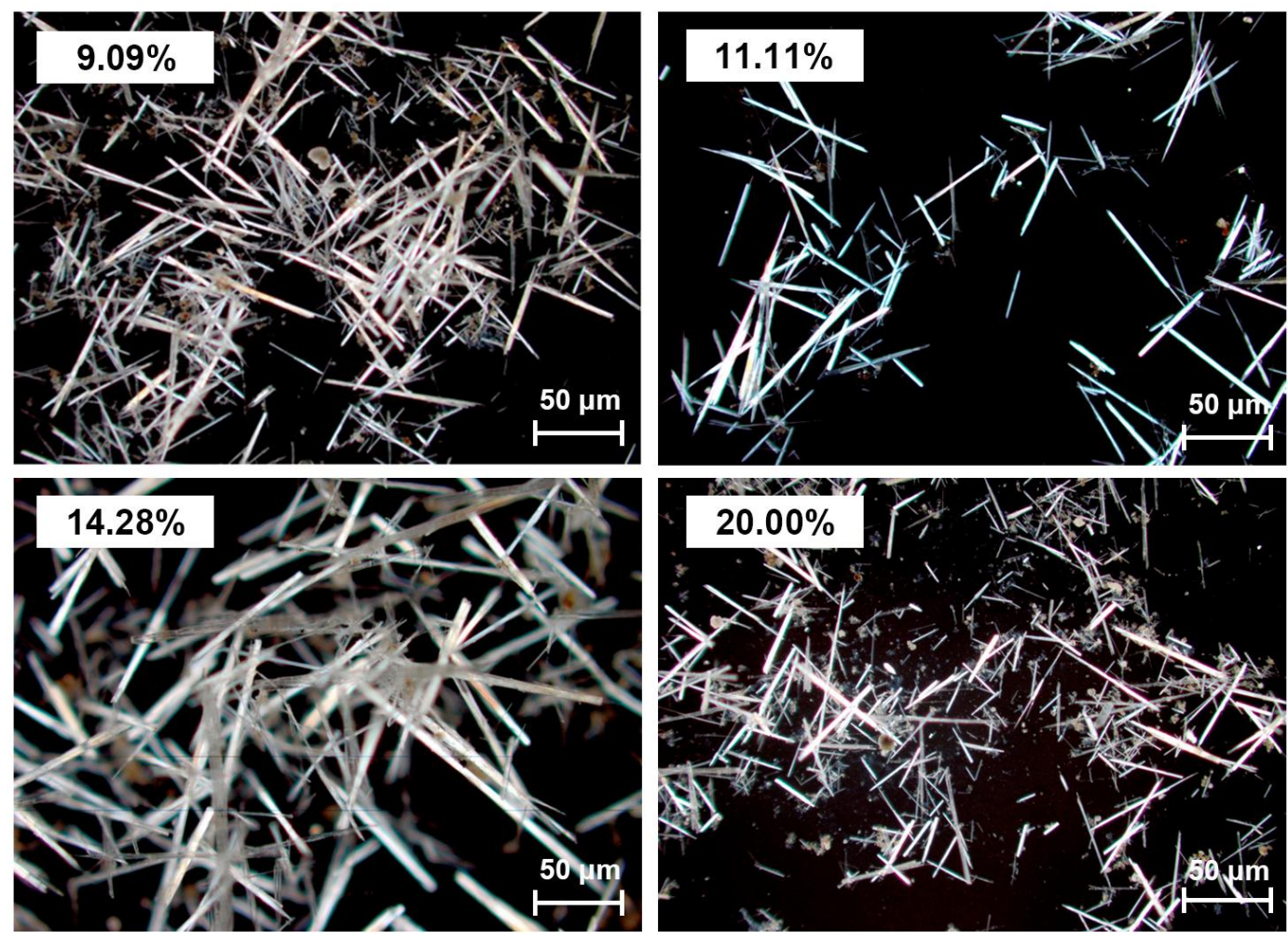

Figure 5 Microscope images of CSW with different slurry concentrations.

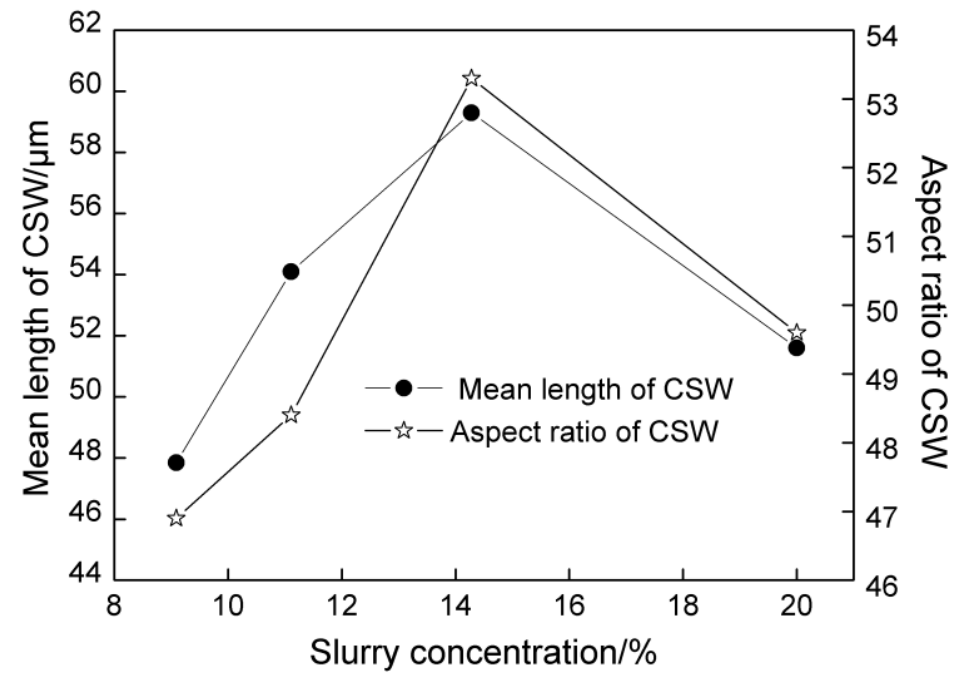

Figure 6 Mean length and aspect ratio of CSW as a function of slurry concentration. 
The explanation of this finding could be attributed to the relative supersaturation variation caused by the slurry concentration $(\sigma)$, which was expressed as

$$
=\frac{C \quad C}{C}=\frac{C}{C} 1
$$

where $C$ is the total concentration of the calcium sulfate, and $C^{*}$ is the equilibrium concentration of the calcium sulfate. Therefore, the growth rate of CSW $(R)$ could be defined as

$$
R=\frac{q}{\rho_{\mathrm{C}}} \sigma=\frac{q}{\rho_{\mathrm{C}}}\left(\frac{C}{C^{*}}-1\right)
$$

where $q$ is the supply coefficient of calcium sulfate, and $\rho_{\mathrm{C}}$ is the density of CSW. A directly proportional relationship between the growth rate of CSW and slurry concentration was obtained, which meant that relatively higher concentration slurry causes more severe surpersaturation improving the CSW growth. However the viscosity of the slurry increased with concentration elevation as well, which could inhibit the ions diffraction from the bulk of solution to the surface of whisker. Therefore, when the slurry concentration was too high like $20.00 \%$ the development of CSW went bad.

\subsection{Effect of additives}

Although the needle-like CSW (tens of microns long) was prepared by optimizing particle size and slurry concentration, the morphology may be further improved by adding additives. Various additives work in different 
ways, such as some additives could change the equilibrium saturation of specie in aqueous, and some could absorb

on the specific surface of crystal changing the crystallization rate in a certain direction. Based on the contributions researchers made before [14-24], some additives including magnesium chloride, citric acid and SDBS was selected and employed for CSW fabrication in hydrothermal condition under the obtained optimal process conditions $\left(D_{50}=6.15 \mu \mathrm{m}\right.$, slurry concentration was $\left.14.28 \%\right)$, and the synergistic effect was studied as well.

\subsubsection{Magnesium chloride}

Some magnesium chloride was added in the CSW preparation process, and the concentration (mass fraction of FGD gypsum) investigated in this section were $0 \%, 2 \%, 3 \%$, and $4 \%$. The relationship between the mean length and aspect ratio of CSW and the magnesium chloride concentration can be seen in Fig. 7. Experimental results indicated that both mean length and aspect ratio sharply decreased with magnesium chloride dosage in the slurry while the diameter had a slight increase, demonstrating that the employment of this additive significantly worsened the CSW morphology. The explanation of this phenomenon was proposed as that preferential adsorption of $\mathrm{Mg}^{2+}$ at active growth sites on transverse surface of long axis, so the growth rate of this direction was inhibited [18], resulting in the crystal shape finally developed to be columnar-like rather than needle-like. 


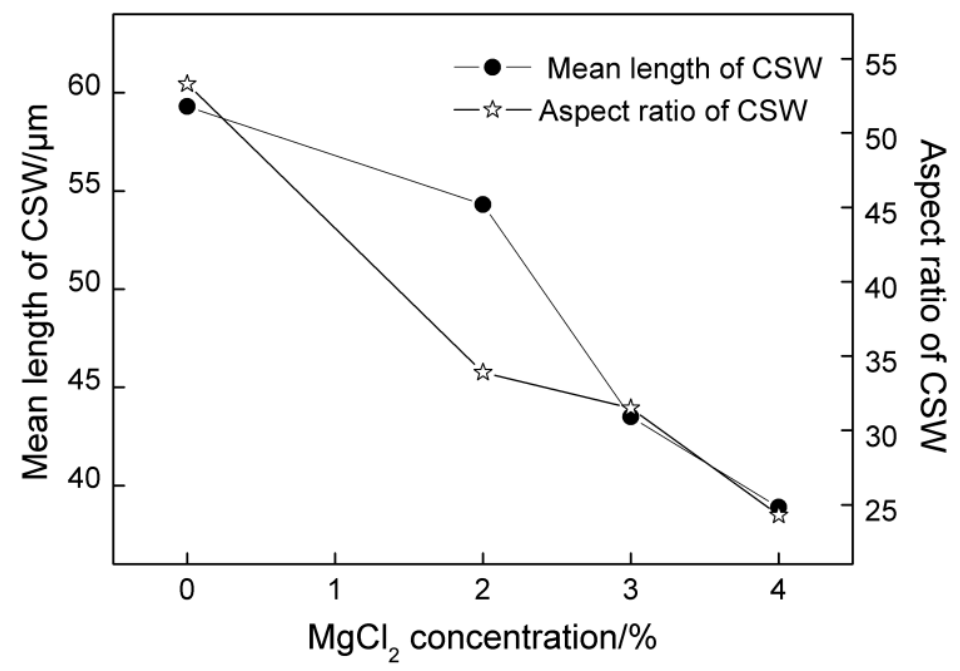

Figure 7 Mean length and aspect ratio of CSW as a function of $\mathrm{MgCl}_{2}$ concentration.

Yang et al. [26] reported that the growth rate of calcium sulfate crystal could be improved by adding $\mathrm{Mg}^{2+}$ in low concentration slurry, and the author attributed this variation to the interaction between $\mathrm{Mg}^{2+}$ and $\mathrm{SO}_{4}^{2-}$ to form neutral specie like $\mathrm{MgSO}_{4}$, which leaded to a initial supersaturation enhancing effects [20]. However, this facilitation of $\mathrm{Mg}^{2+}$ cannot play a lead role in high concentration slurry, because the slight supersaturation change caused by a small quantity of $\mathrm{Mg}^{2+}$ is negligible when the concentration of $\mathrm{SO}_{4}^{2-}$ is high enough [21]. Therefore, the researchers confirmed that the employment of $\mathrm{Mg}^{2+}$ in low concentration slurry was advantageous to obtain well-crystallized calcium sulfate, while no positive effects could be found if this metallic ion was used in high concentration slurry. In the current study, high concentration slurry was already demonstrated to be helpful to prepare needle-like CSW, so the single magnesium chloride additive was proved to be useless for morphology improvement. 


\subsubsection{Citric acid}

Barely research focused on the effects of citric acid on CSW has been carried out. Some hydrothermal tests with

various citric acid concentrations for CSW preparation was conducted in this work to investigate the function of this

organic additive on morphology. Figure 8 shows the change of mean length and aspect ratio of CSW with citric acid concentration from $0 \%$ to $0.60 \%$. Results indicated that the mean length increased from $59 \mu \mathrm{m}$ to $66 \mu \mathrm{m}$ and the aspect ratio increased from 53 to 59 when citric acid concentration was $0.3 \%$, but they both significantly went down if more dosage was added. Complex reaction could happen between citric acid and $\mathrm{Ca}^{2+}$ to form complex compound promoting calcium sulfate hydrolysis, so the growth process of CSW was slightly accelerated when a few citric acid like $0.3 \%$ was employed. Taking into account of the crystal structures of citric acid and CSW, the distance between two of the oxygen ions of the two carboxylic group of the molecule of citric acid is exactly the same as the distance between two neighboring calcium ions on some CSW faces. Thus the severe adsorption of citric acid on CSW surface results in a retardation of $\mathrm{Ca}^{2+}$ and $\mathrm{SO}_{4}^{2-}$ deposit on crystal, and, as a result, the CSW growth decelerates. This absorption effect of citric acid on calcium sulfate crystal surfaces in crystal growth process was proved to be reasonable by Peng et al. [22]. Therefore, similar to the magnesium chloride, the additive of citric acid could inhibit the growth rate of CSW during the hydrothermal synthesis process, so the CSW morphology was changed consequently. 


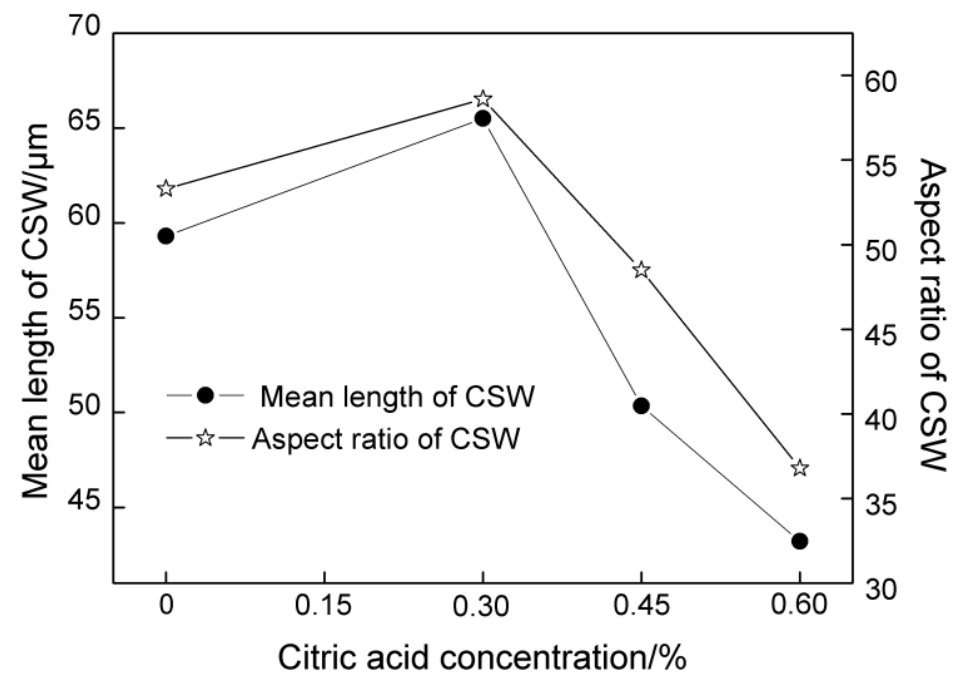

Figure 8 Mean length and aspect ratio of CSW as a function of citric acid concentration.

\subsubsection{SDBS}

SDBS that is known as an effective dispersant was employed in hydrothermal synthesis process, and the trend

of mean length and aspect ratio with SDBS concentration from $0 \%$ to $0.6 \%$ was investigated. Experimental results

showed a slight increase when the SBDS concentration was $0.2 \%$ but did not experience any notable changes with

the SDBS concentration elevation. Microscope images of CSW products obtained from different SDBS

concentration tests $(0 \%, 0.2 \%, 0.4 \%$, and $0.6 \%)$ are given in Fig. 9 to show their apparent morphology change. It

could be found that CSW became finer and more uniform when some SDBS was employed. However, coinciding

with the results obtained from mean length and aspect ratio calculation, the apparent morphology of CSW exhibited

no obvious change when the SDBS concentration went to $0.4 \%$ or $0.6 \%$. In the hydrothermal synthesis process, the

viscosity of slurry would increase with duration because of the elevation of nucleation number of CSW. SDBS, as an 
anionic surfactant with an excellent dispersing performance, could effectively control the viscosity variation, so a

sharp change of ionic diffusion rate in solution could be prevented and the probability of second nucleating of CSW

can be reduced. For these reasons, the apparent morphology of CSW was improved, but no significant correlation

with SDBS concentration was found.
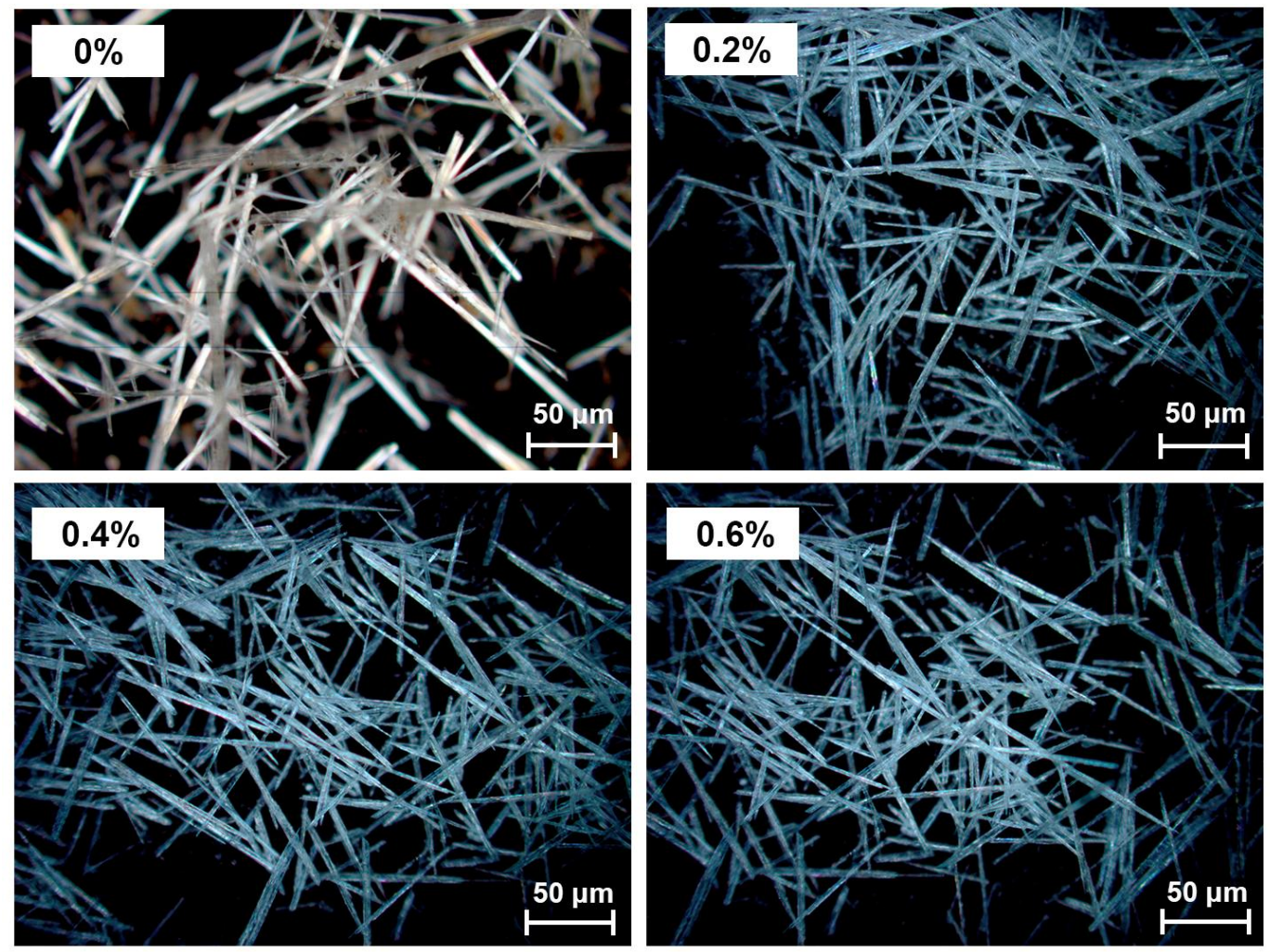

Figure 9 Microscope images of CSW with different SDBS concentrations.

\subsubsection{Synergistic effect}

Inspired by the findings obtained above, morphology improvement of CSW by adding multi-additives like

magnesium chloride-SDBS or citric acid-SDBS may be achieved if the SDBS could change the absorption behavior 
of magnesium chloride and citric acid. Synergistic effect of various magnesium chloride concentrations ( $0 \%, 2 \%$,

$3 \%$, and $4 \%$ ) and a constant SDBS concentration (0.2\%) on the mean length and aspect ratio of CSW was investigated, and the experimental data is given in Fig. 10. A similar trend was observed with the results of SDBS-free tests. The mean length and aspect ratio went down with the magnesium chloride concentration and some stubby CSW was produced. It was demonstrated that the SDBS was useless to change negative effects of magnesium chloride, and, moreover, the dispersing performance of SDBS cannot show up in the presence of magnesium chloride. Therefore, magnesium chloride should be avoided in this CSW preparation process.

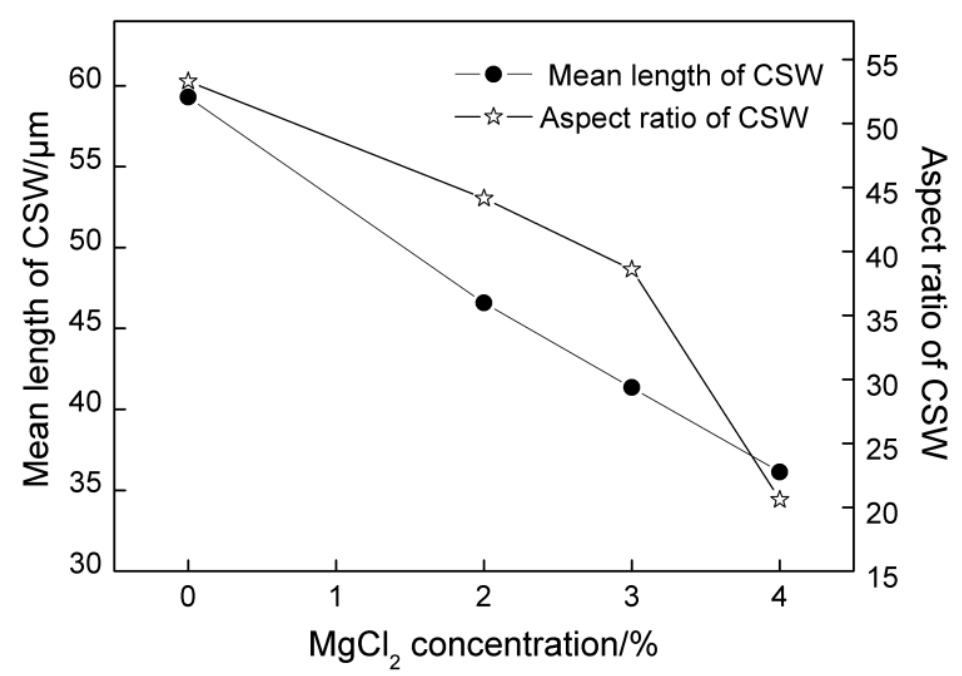

Figure 10 Mean length and aspect ratio of CSW as a function of $\mathrm{MgCl}_{2}$ concentration with $0.2 \%$ of SDBS.

A batch of tests with different citric acid concentrations $(0 \%, 0.30 \%, 0.45 \%$, and $0.60 \%)$ and constant SDBS concentration $(0.2 \%)$ was carried out. With reference to the results in Fig. 11, it was found that when the citric acid 
concentration was $0.3 \%$ the mean length of CSW increased from $59 \mu \mathrm{m}$ to $71 \mu \mathrm{m}$ and the aspect ratio of CSW increased from 53 to 66 , showing a growth rate more than $20 \%$. With a constant SDBS concentration of $0.2 \%$, the two indexes of CSW product cannot have a further elevation when the citric acid concentration went higher, which was coincident with the SDBS-free results. However, comparing with data obtained from SDBS-free tests, the mean length and aspect ratio of CSW prepared from citric acid-SDBS tests were both increased more than $20 \%$ when citric acid concentration reached $0.45 \%$ and $0.60 \%$. The reason could be attributed to the absorption of citric acid on crystal surface was weakened since the dispersive effect of SDBS.

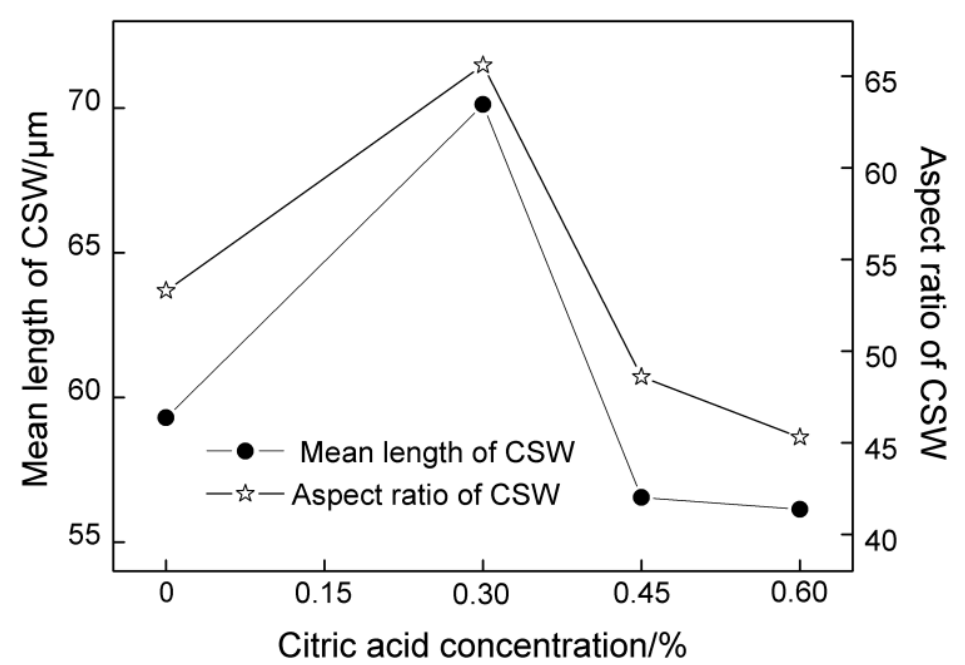

Figure 11 Mean length and aspect ratio of CSW as a function of citric acid concentration with $0.2 \%$ of SDBS.

SEM analysis was conducted to observe the surface morphology of CSW, and the images of products obtained 
found in the product from non-additive test, and the particle size and crystal shape of various CSW showed a distinct

difference. When multi-additives of $0.3 \%$ citric acid-0.2\% SDBS were employed, the apparent morphology of CSW

was significantly improved, and the particle size and crystal shape became uniform. XRD pattern of final product

obtained from optimum conditions is given in Fig. 13, showing the principal phase to be HH whisker with a high

crystallinity. Additionally, three strong diffraction peaks of HH whisker represented three crystal surfaces of (200),

(220), and (400) that were all parallel to c axis demonstrating the CSW grew along this axis. Experimental findings

of this study proved that the utilization of citric acid and SDBS in hydrothermal synthesis process effectively

promoted the development of CSW, and needle-like crystal with high length and well morphology was prepared.
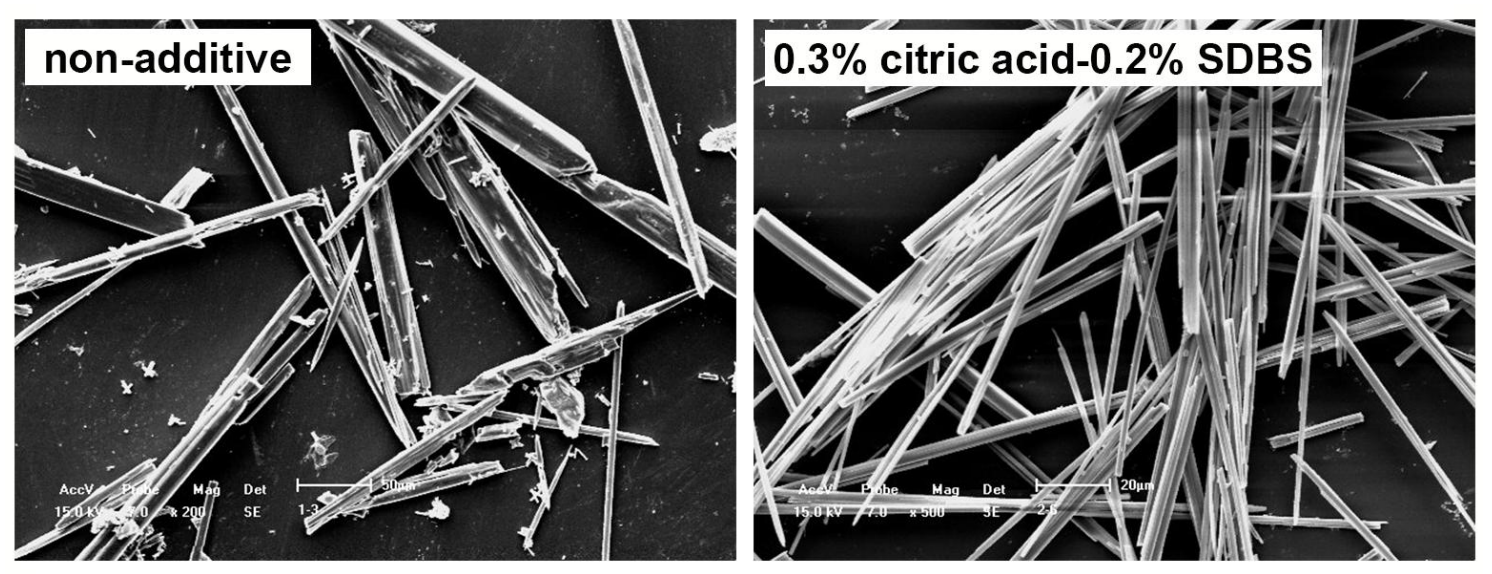

Figure 12 SEM images of CSW obtained from non-additive test and $0.3 \%$ citric acid- $0.2 \%$ SDBS. 


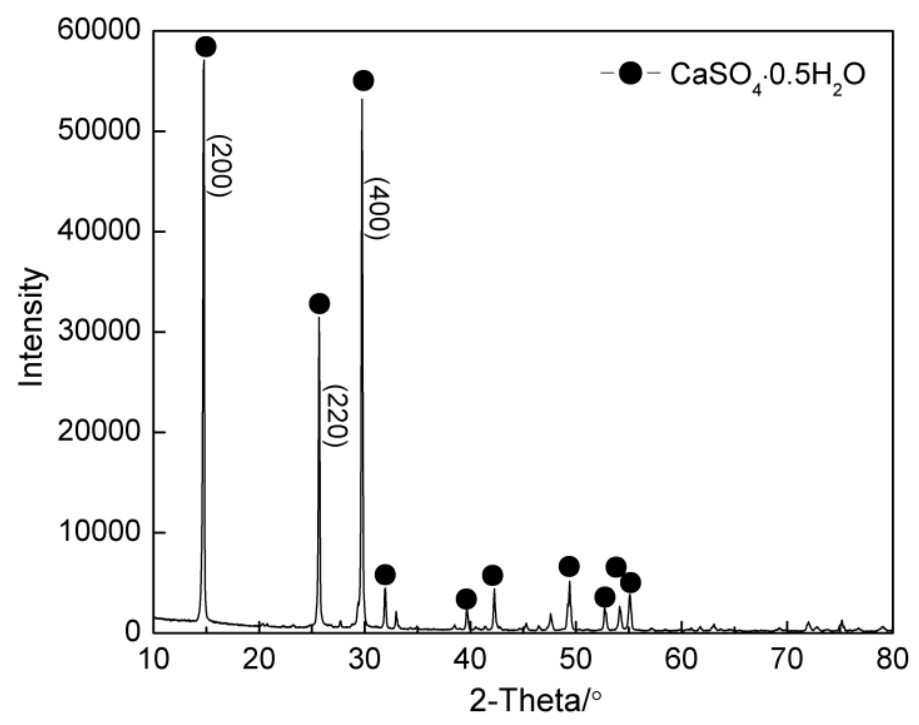

Figure 13 XRD pattern of CSW product.

\subsection{Comparison of CSWs prepared from different raw materials}

Some CSW was prepared from magnesium sulfate and calcium chloride anhydrous at the same conditions. Fig.

14 shows the SEM images of CSWs produced from different raw materials, and a difference in morphology was

clearly observed. The length of the two CSWs was in the same scale (dozens of micrometer), but a smaller diameter

CSW was obtained in FGD gypsum tests meaning better mechanical properties may be achieved. Furthermore, the surface of CSW manufactured from chemical reagents was quite smooth, while some grooves existed on the surface of FGD gypsum prepared CSW increasing the specific surface area, which would influence the subsequent surface modification process. Therefore, published studies on surface modification of CSW prepared from other raw 
materials may not be useful to explain the modification behavior of CSW prepared from FGD gypsum. In order to

fill this research gap, a surface modification studies on the obtained CSW was conducted and reported in the other

part of this work [25].
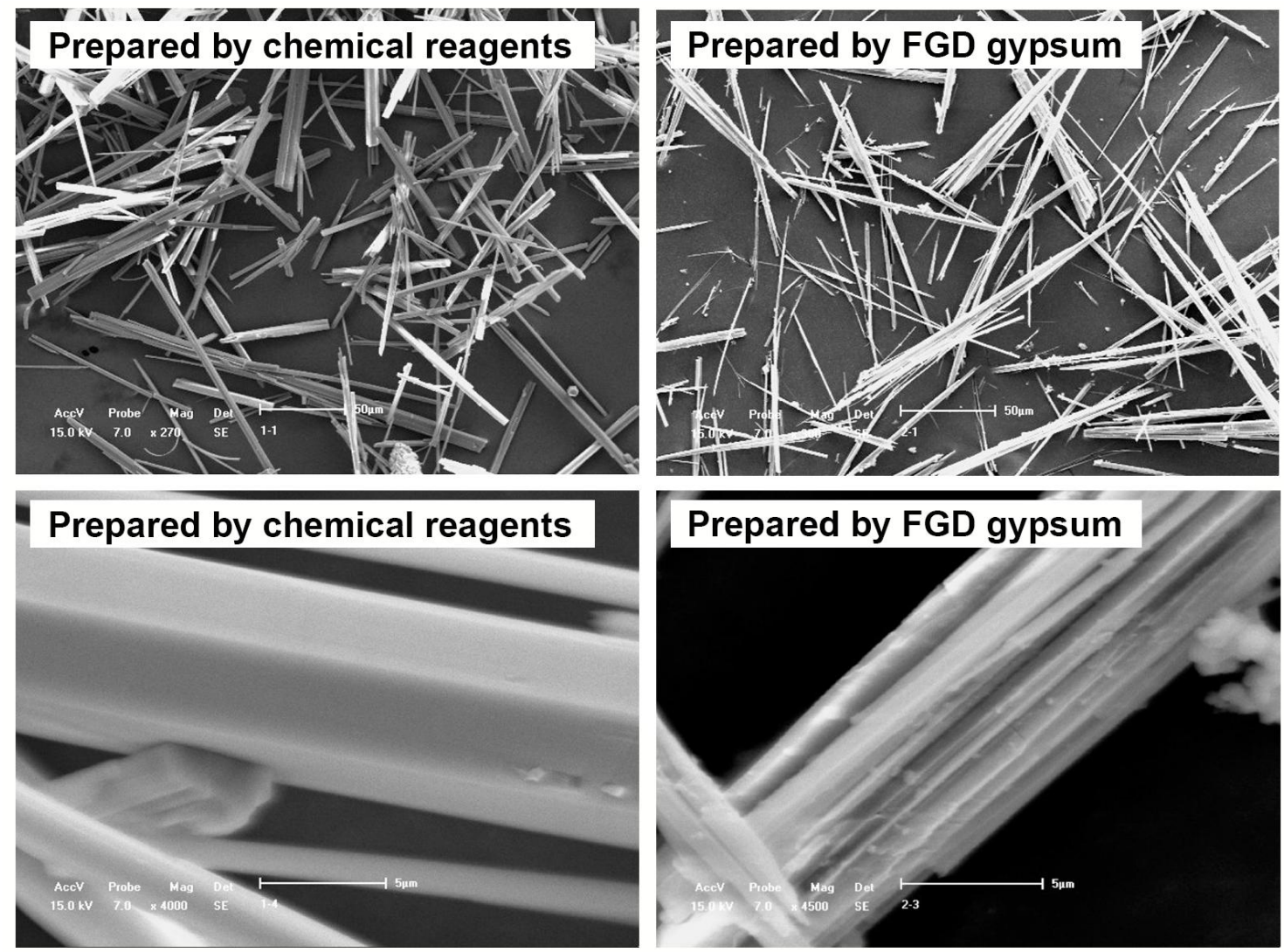

Figure 14 SEM images of CSW prepared from different raw materials.

4. CONCLUSIONS

Needle-like CSW was prepared from FGD gypsum by hydrothermal synthesis process. The effects of particle 
size of FGD gypsum, slurry concentration, and additives on CSW were investigated in the current work.

Experimental results showed that fine particle size of FGD gypsum and moderately high slurry concentration were beneficial for CSW nucleation and growth. Magnesium chloride, citric acid and SDBS were employed as additives for improving CSW morphology. It was found that magnesium chloride inhibited the crystal growth reducing the mean length and aspect ratio of CSW, and SDBS dispersant exhibited no alleviated effect to this phenomenon. A small quantity of citric acid or SDBS was demonstrated to be helpful to improve the CSW morphology, and a further improvement could be achieved when the two additives was simultaneously used. When citric acid concentration was $0.3 \%$ and SDBS concentration was $0.2 \%$, the mean length of CSW reached $71 \mu \mathrm{m}$ and the aspect ratio reached 66, which were both higher than $20 \%$ relative to that of the CSW prepared from additive-free test. Moreover, by using this multi-additives, crystal defect of CSW were significantly decreased, and the particle size and crystal shape became more uniform. Additionally, CSW prepared from industrial FGD was much finer than that from chemical reagents.

The other part of this study focuses on improving hydrophobic property of the obtained CSW by surface modification treatment, and the reasonable modifying conditions are revealed [25]. 


\section{REFERENCE}

1 Guan, B.H., Fu, H.L., Yu, J., Jiang, G.M., Kong, B., Wu, Z., "Direct transformation of calcium sulfite to $\alpha$-calcium sulfate hemihydrate in a concentrated $\mathrm{Ca}-\mathrm{Mg}-\mathrm{Mn}$ chloride solution under atmospheric pressure", Fuel, 90(1), 36-41 (2011).

2 Álvarez-Ayuso, E., Querol, X., Tomás, A., "Environmental impact of a coal combustion-desulphurisation plant: abatement capacity of desulphurisation process and environmental characterization of combustion by-products”, Chemosphere, 65(11), 2009-2017 (2006).

3 Córdoba, P., "Status of Flue Gas Desulphurisation (FGD) systems from coal-fired power plants: Overview of the physic-chemical control processes of wet limestone FGDs", Fuel, 144, 274-286 (2015).

4 Tzouvalas, G., Rantis, G., Tsimas, S., “Alternative calcium-sulfate-bearing materials as cement retarders: Part II: FGD gypsum”, Cement Concrete Res., 34(11), 2119-2125 (2004).

5 Chandara, C., Azizli, K.A.M., Ahmad, Z.A., Sakai, E., "Use of waste gypsum to replace natural gypsum as set retarders in portland cement”, Waste Manage., 29(5), 1675-1679 (2009).

6 Miao, M., Feng, X., Wang, G.L., Cao, S.M., Shi, W., Shi, L.Y., "Direct transformation of FGD gypsum to calcium sulfate hemihydrate whiskers: Preparation, simulations, and process analysis”, Particuology, 19(2), 53-59 (2015).

7 Feldmann, T., Demopoulos, G.P., "The crystal growth kinetics of alpha calcium sulfate hemihydrate in concentrated $\mathrm{CaCl}_{2}-\mathrm{HCl}$ solutions", J. Cryst. Growth, 351(1), 9-18 (2012).

8 Papageorgiou, A., Tzouvalas, G., Tsimas, S., "Use of inorganic setting retarders in cement industry", Cement and Concrete Comp., 27(2), 183-189 (2005).

9 Zhu, Z.C., Xu, L., Chen, G.A., Li, Y.L., "Optimization on tribological properties of aramid fibre and $\mathrm{CaSO}_{4}$ whisker reinforced non-metallic friction material with analytic hierarchy process and preference ranking 
organization method for enrichment evaluations", Mater. Des., 31(1), 551-555 (2010).

10 Freyer, D., Voigt, W., "Crystallization and Phase Stability of $\mathrm{CaSO}_{4}$ and $\mathrm{CaSO}_{4}$-Based Salts", Monatsh. Chem., 134(5), 693-719 (2003).

11 Luo, K.B., Li, C.M., Xiang, L., Li, H.P., Ning P., "Influence of temperature and solution composition on the formation of calcium sulfates", Particuology, 8(3), 240-244 (2010).

12 Wang, X., Yang, L.S., Zhu, X.F., Yang, J.K., "Preparation of calcium sulfate whiskers from FGD gypsum via hydrothermal crystallization in the $\mathrm{H}_{2} \mathrm{SO}_{4}-\mathrm{NaCl}-\mathrm{H}_{2} \mathrm{O}$ system", Particuology, 17(6), $42-48$ (2014).

13 Yang, L.S., Wang, X., Zhu, X.F., Du, L.Z., "Preparation of calcium sulfate whisker by hydrothermal method from flue gas desulfurization (FGD) gypsum”, Applied Mechanics and Materials, 268-270, 823-826 (2013).

14 Cölfen, H., Qi, L.M., Mastai, Y., Börger, L., "Formation of unusual 10-petal $\mathrm{BaSO}_{4}$ structures in the presence of a polymeric additive”, Cryst. Growth Des., 2(3), 191-196 (2002).

15 Wang, C., "Control the polymorphism and morphology of calcium carbonate precipitation from a calcium acetate and urea solution", Mater. Lett., 62(16), 2377-2380 (2008).

16 Yang, C., Song, X.F., Sun, S.Y., Sun, Z., Yu, J.G., "Effects of sodium dodecyl sulfate on the oriented growth of nesquehonite whiskers", Adv. Powder Technol., 24(3), 585-592 (2003).

17 Guan, B.H., Yang, L.C., Wu, Z.B., Shen, Z.X., Ma, X.F., Ye, Q., "Preparation of $\alpha$-calcium sulfate hemihydrate from FGD gypsum in $\mathrm{K}, \mathrm{Mg}$-containing concentrated $\mathrm{CaCl}_{2}$ solution under mild conditions”, Fuel, 88(7), $1286-1293$ (2009).

18 Hamdona, S.K., Hadad, U.A.A., "Crystallization of calcium sulfate dihydrate in the presence of some metal ions", J. Cryst. Growth, 299(1), 146-151 (2007).

19 Rashad M.M., Mahmoud M.H.H., Ibrahim I.A., Abdel-Aal E.A., "Crystallization of calcium sulfate dihydrate under simulated conditions of phosphoric acid production in the presence of aluminum and magnesium ions", J. Cryst. Growth, 267(1-2), 372-379 (2004). 
20 Wang, L., Ma, J.H., Guo, Z.W., Dong, B.S., Wang, G.M., "Study on the preparation and morphology of calcium sulfate whisker by hydrothermal synthesis method", Mater. Sci. Technol., 14(6), 626-629 (2006). (In Chinese)

21 Yang, N., Xiao, H.N., Guo, W.M., “Additives-assisted hydrothermal synthesis of calcium sulfate whisker and its growth mechanism", Journal of the Chinese Ceramic Society, 42, 539-544 (2014). (In Chinese)

22 Peng, J.H., Qu, J.D., Wu, L., Zhang, J.X., "Effect of critic acid on crystalline habit and crystal morphology of dehydrate", Journal of Southeast University (Natural Science Edition), 34(3), 356-360 (2004). (In Chinese)

23 Prisciandaro, M., Lancia, A., Musmarra, D., "The retarding effect of citric acid on calcium sulfate nucleation kinetics", Ind. Eng. Chem. Res., 42(25), 6647-6652 (2003).

24 Mao, X.L., Song. X.F., Lu, G.M., Xu, Y.X., Sun, Y.Z., Yu, J.G., "Effect of additives on the morphology of calcium sulfate hemihydrate: Experimental and molecular dynamics simulation studies”, Chem. Eng. J., 278, 320-327 (2015).

25 Liu, C.J., Zhao, Q., Wang, Y.G., Shi, P.Y., Jiang, M.F., "Surface modification of calcium sulfate whisker prepared from flue gas desulfurization gypsum”, Appl. Surf. Sci., 360, 263-269 (2016).

26 Yang, L.C., Wu, Z.B., Guan, B.H., Fu, H.L., Ye, Q.Q., "Growth rate of a-calcium sulfate hemihydrate in K-Ca-Mg-Cl-H ${ }_{2} \mathrm{O}$ systems at elevated temperature”, J. Cryst. Growth, 311, 4518-4524 (2009). 

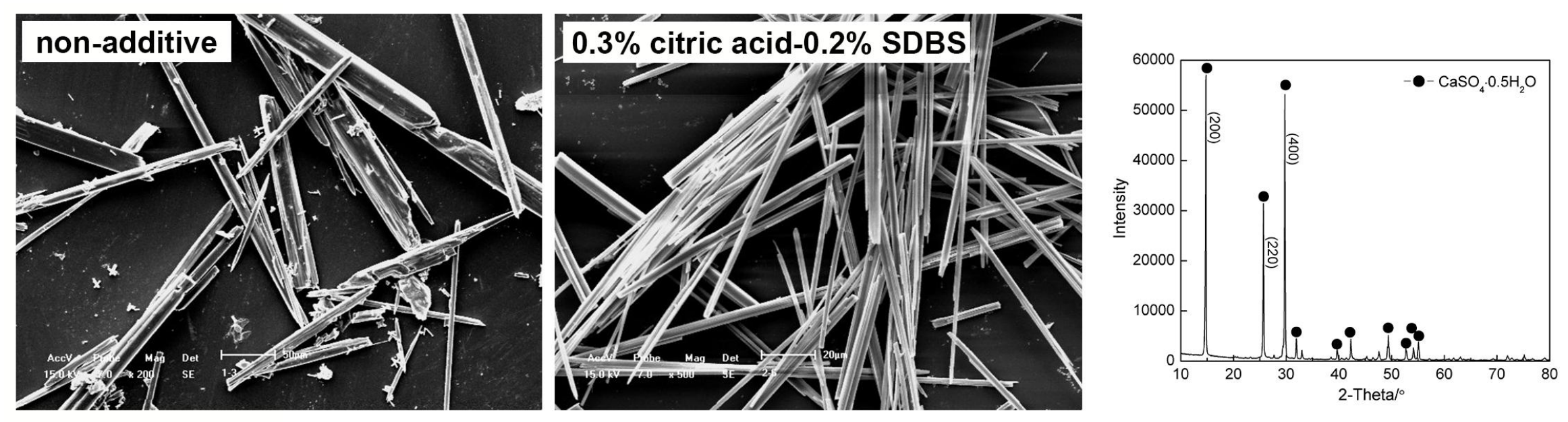